\title{
Sex differences in the effects of Auricularia auricula-judae ethanolic extracts on the life span of Drosophila melanogaster during stress and non-stress conditions
}

\author{
JC James N. Romero ${ }^{1}$, Ma. Rosario Rufina E. Ramos ${ }^{1}$, Ourlad Alzeus G. Tantengco ${ }^{2}$, Paul Mark B. Medina ${ }^{2 *}$ \\ 'Department of Biology, College of Arts and Sciences, University of the Philippines Manila, Manila, Philippines. \\ ${ }^{2}$ Biological Models Laboratory, Department of Biochemistry and Molecular Biology, College of Medicine, University of the Philippines Manila, Manila, \\ Philippines.
}

\begin{tabular}{l}
\hline ARTICLE INFO \\
\hline Received on: $15 / 02 / 2018$ \\
Accepted on: $22 / 03 / 2018$ \\
Available online: $30 / 11 / 2018$ \\
\\
\hline Key words: \\
Auricularia auricula-judae, \\
life span, heat stress, energy \\
deprivation, DPPH assay.
\end{tabular}

\section{INTRODUCTION}

Aging is a biological phenomenon manifested by the progressive decline of physiological integrity and function within living organisms over time. This decline associates aging with the progressive increase in mortality and age-related diseases such as cardiovascular disorder and diabetes (Iliadi and Boulianne, 2010). Aging is natural and unavoidable, but experimental research studies have determined that there are genetic and non-genetic factors altering the normal aging process (Magwere et al., 2004). The fruit fly, Drosophila melanogaster, is one of the most popular

\section{*Corresponding Author}

Paul Mark B. Medina, Biological Models Laboratory, Department of Biochemistry and Molecular Biology, College of Medicine, University of the Philippines Manila, Manila, Philippines.

E-mail:pmbmedina@post.upm.edu.ph model organisms in aging research because of its short life span and low-maintenance requirements. Its genome was fully sequenced with more than $50 \%$ homologs in humans. Almost $75 \%$ of known human genetic disorders have fly homologs (Sun et al., 2013).

Dietary interventions have been shown to increase life span of various animal species including flies. Sex differences in the life span of male and female flies have been reported which may be attributed to the fundamental differences in the amount of energy needed by both sexes (Hunt et al., 2011; Magwere et al., 2004; Velasco and Medina, 2014). Natural products with high antioxidant levels have been reported to increase the life span based on studies on D. melanogaster (Kumar et al., 2015; Lin et al., 2014; Rawal et al., 2014). Mushrooms, harboring numerous bio-active components, appear to be a promising anti-aging agent (Azizur Rahman et al., 2017). Edible mushrooms are good sources of antioxidants (Acharya et al., 2005; Boonsong et al., 2016; Luo et al., 2009). Auricularia auricula-judae, locally known as "tenga 
ng daga," is a popular edible mushroom in the Philippines. It is widely used in Chinese folk medicine for dysentery, numbness, and as a topical treatment of a sore throat, conjunctivitis, and tonsillitis (Kadnikova et al., 2015). Several studies have reported high antioxidant activity of $A$. auricula-judae (Asatiani et al., 2007; Kho et al., 2009; Zou et al., 2015). Other study shows that an extract from $A$. auricula extended the life span of $D$. melanogaster in a dose- and sex-dependent manner (Huang et al., 2011). It was also reported to exhibit many medicinal properties such as antitumor activity, blood pressure regulation, and a decrease in the concentration of lipid and cholesterol in the blood (Wasser and Weis, 1999).

Auricularia auricula-judae has been a part of the diet of Filipinos, especially among some indigenous tribes. Thus, we explored the potential effects of $A$. auricula-judae crude ethanolic extracts in life span using $D$. melanogaster. We investigated the antioxidant activities of wild and cultivated $A$. auricula-judae and determined their effects in the life span of adult $D$. melanogaster in the presence and absence of environmental stress such as heat stress and starvation stress.

\section{MATERIALS AND METHODS}

\section{Preparation of mushroom extracts}

Wild A. auricula-judae was collected from Hermosa, Bataan, Philippines, while cultivated $A$. auricula-judae was bought from a commercial establishment in Chinatown, Manila. Voucher specimens were submitted to the Botany Department of the National Museum of the Philippines for taxonomic identification.

Wild A. auricula-judae was washed with water three times: twice with tap water then once with distilled water. Then, they were chopped to smaller pieces, dried under shade at room temperature, and powdered using an electric blender. The cultivated $A$. auricula-judae was already dry when purchased and was directly pulverized using an electric blender.

The resulting powders were then subjected to ethanolic extraction using 95\% ethanol (Belman Laboratories; Philippines) as a solvent. One hundred grams of the wild and cultivated mushroom samples were then soaked separately in $200 \mathrm{ml}$ of $95 \%$ ethanol for 72 hours. Each mixture was filtered using Whatman No. 1 filter paper, then the filtrate was subjected to a rotary evaporator (Rotavap R-200, Buchii, Switzerland) at $38^{\circ} \mathrm{C}$. The resulting extracts were collected in an evaporating dish, then labeled as wild Auricularia ethanolic extract (WAEE) and cultivated Auricularia ethanolic extract (CAEE), respectively. The extracts were then subjected to a $40^{\circ} \mathrm{C}$ water bath inside a fume hood for a few days or until the complete evaporation of the solvent. Both WAEE and CAEE were stored inside the refrigerator at $4{ }^{\circ} \mathrm{C}$.

\section{Preparation of fly media}

The sweet potato yeast agar media consisted of $10 \mathrm{~g}$ of yeast (Saf-Instant Gold, Lessafre, France), $15.4 \mathrm{~g}$ of agar (Agar Powder, Titan Biotech Limited, India), and 1,000 $\mathrm{ml}$ distilled water for every $500 \mathrm{~g}$ of sweet potato, Ipomoea batatas (L.) Lam.

The sweet potato was bought from a local market in Pasay, Philippines and then washed, peeled, and boiled in distilled water until soft and tender. Water was drained and the sweet potato was then mixed with yeast, agar, and distilled water. The resulting mixture was allowed to boil and cool down to $60^{\circ} \mathrm{C}$ before adding $0.08 \%$ propionic acid (Propionic Acid Laboratory Grade, Qualikems, India). The final mixture was placed in their final containers before adding the yeast paste superficially. Yeast paste consisted of 1 part yeast (Saf-Instant Gold, Lessafre, France) and 1 part $0.08 \%$ propionic acid.

\section{Test animals}

Wild-type D. melanogaster was obtained from the Department of Biochemistry and Molecular Biology, College of Medicine, University of the Philippines Manila. The flies were bred according to the protocol established by Velasco and Medina (2014). Flies were age-synchronized by the daily removal of newly emerged flies from a general breeding population and then placed in a fresh new media labeled as Day 0 flies. On Day 3, flies were immobilized and anesthetized in a fly pad using carbon dioxide gas (Alvimco Company Inc., Philippines) and then sorted by sex using a stereomicroscope.

\section{Chronic lethality test}

Chronic lethality test served as an optimization assay in order to determine the sublethal and non-toxic concentrations of the ethanolic extracts of $A$. auricula-judae to be used as a daily food supplementation to the fly media for the experimental setups. Three increments of WAEE and CAEE concentrations were supplemented to the fly media for this assay specifically: $0.1,0.5$, and $1.0 \mathrm{mg} / \mathrm{ml}$. For each concentration of the extract, 20 male flies and 20 female flies were used. Percent survival of the flies was noted every day for 5 days. Observed mortality must be due to the chronic effects ( $>48$ hours) and not as acute effects $(<48$ hours) of the mushroom extracts. A fly was considered dead if it no longer exhibits any movement after some time.

Concentrations with percent survival higher than $90 \%$ were considered sublethal. The highest sublethal concentration was considered as the high dosage. Middle and low experimental dosages were calculated as a 10-fold lower and 100-fold lower of the high dosage, respectively. High, middle and low dosages were used as the experimental concentrations treated as food supplements for the life span assay (LSA), heat stress assay (HSA), and starvation assay (SA).

\section{Life span assay}

Three-day-old flies were anesthetized with $\mathrm{CO} 2$, sorted according to sex, and placed in Drosophila vials in groups of 10 . There were a total of seven setups: one negative control and three experimental setups for each of the WAEE and CAEE. There were 60 flies per setup: 30 males and 30 females. Each group of 10 flies was placed in a Drosophila vial with the corresponding breathable plug (Drosophila vials, Biologix, USA). The setup was maintained at room temperature and the mortality per vial was recorded daily until all flies were dead. The flies were carefully transferred to newly prepared vials every 3 days until the end of their lifetime.

\section{Heat stress assay}

The HSA was a modified LSA. All Drosophila vials were placed inside a $37^{\circ} \mathrm{C}$ incubator 1 hour every day. Mortality per vial was recorded every day until all the flies died. Flies were also transferred to new media every 3 days. 


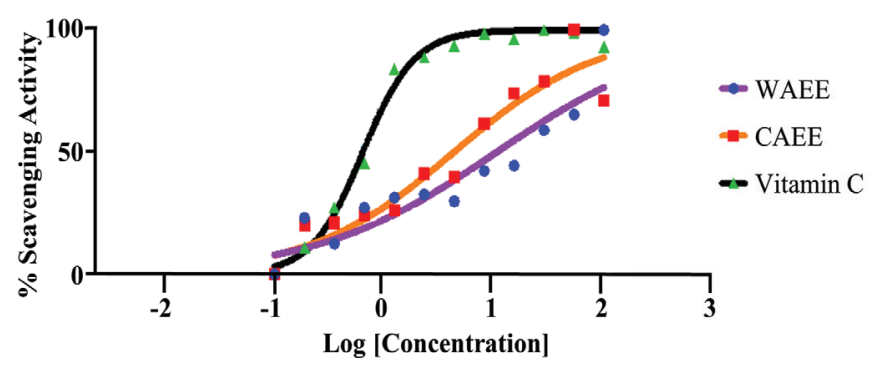

Figure 1. Dose-response curves of normalized values of $\%$ activity $v s$. concentration of $A$. auricula-judae ethanolic extracts and a commercial antioxidant (Vitamin C).

\section{Starvation assay}

The SA was also a modified LSA. Instead of transferring the flies to fresh media after the first 3 days, the flies were placed in a Drosophila vial containing only moist cotton. All of the Drosophila vials were kept in room temperature. Hourly mortality per vial was counted until all flies were dead.

\section{DPPH free radical scavenging assay}

The total antioxidant activities of WAEE and CAEE were measured using the 2,2-diphenyl-1-picrylhydrazyl (DPPH) Assay. The DPPH radical (DPPH, Sigma-Aldrich, Singapore) used was at $0.2 \mathrm{mM}$ in $95 \%$ ethanol. Aliquots of the WAEE and CAEE dissolved in dimethyl sulfoxide were plated out in triplicates in a 96-well microtiter plate.Ethanol was used as a negative control, while vitamin $\mathrm{C}$ was used as a positive control. The ethanolic DPPH solution was added to alternating columns of the test samples. The plate was shaken for 2 minutes and incubated for 20 minutes in darkness at $37^{\circ} \mathrm{C}$ in a water bath. The difference in absorbance values was obtained using a spectrophotometer (Bio Rad Microplate Reader 550, Bio Rad, China) at $517 \mathrm{~nm}$. The percentage of scavenging activity on DPPH radicals was calculated using the following formula

$$
\% \mathrm{SA}=\left[\frac{\left(\mathrm{Abs}_{\text {sample }}-\mathrm{Abs}_{\text {control }}\right)}{\mathrm{Abs}_{\text {control }}}\right] \times 100
$$

The effective concentration (EC50), the concentration of the sample scavenging $50 \%$ of the initial DPPH radical, can be calculated by interpolating the $\left[\left(\mathrm{Abs}_{\text {sample }}-\mathrm{Abs}_{\text {control }}\right]\right.$ into a calibration line generated by the absorbance of DPPH at different concentrations.

\section{Statistical analysis}

Standard survival curves were constructed using the online application for survival analysis 2 (OASIS 2) (Han et al., 2016). The average life span of flies in control and treatment groups was calculated and analyzed using the Wilcoxon-Breslow-Gehan Test. p value of 0.05 and below was considered significant. $p$ value of 0.001 was considered as highly significant. One-way analysis of variance with Tukey's post-hoc analysis was done using IBM SPSS Statistics Version 21.0 to determine the difference in the antioxidant activity of the crude extracts.

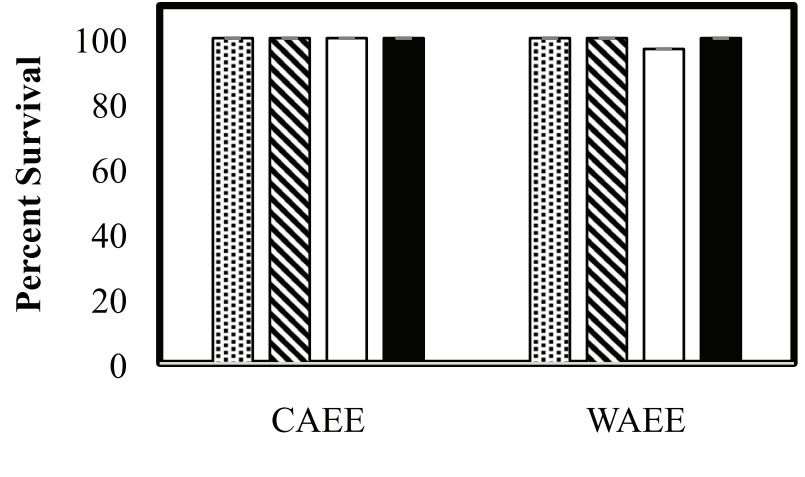

\section{Crude Extracts}

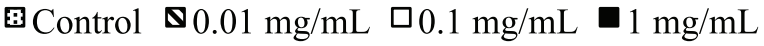

Figure 2. Survival rates of D. melanogaster supplemented with CAEE and WAEE during the chronic lethality assay.

\section{RESULTS AND DISCUSSION}

\section{DPPH free radical scavenging assay}

Results show that CAEE neutralizes DPPH radicals more than WAEE but less effectively than does Vitamin C (Fig. 1). Using the DPPH free radical assay, the EC50 values were 16.64 $\mu \mathrm{g} / \mathrm{ml}$ for WAEE, $6.20 \mu \mathrm{g} / \mathrm{ml}$ for CAEE, and $0.76 \mu \mathrm{g} / \mathrm{ml}$ for Vitamin C. Both WAEE and CAEE exhibited high antioxidant activity but not significantly comparable to that of Vitamin $\mathrm{C}$ $(p<0.001)$. There was an eight-fold decrease in half-maximal effective concentration for CAEE and a 22-fold decrease for WAEE when compared with Vitamin C. This means, the cultivated A. auricula-judae has a higher antioxidant activity compared with wild $A$. auricula-judae.

\section{Chronic lethality test}

All concentrations of WAEE and CAEE used in this study exhibited close to $100 \%$ survival rates for male and female flies after 5 days of supplementation (Fig. 2). WAEE and CAEE were non-lethal to adult male and female flies. Nearly, $1 \mathrm{mg} / \mathrm{ml}$ was assigned as the high dose, $0.1 \mathrm{mg} / \mathrm{ml}$ as the middle dose, and $0.01 \mathrm{mg} / \mathrm{ml}$ as low dose. These sublethal doses were used in all subsequent assays in this study.

\section{Life-span assay}

The LSA revealed that WAEE and CAEE have different effects on the life span of male and female adult flies in the long-term supplementation of extracts. WAEE has neutral effects while CAEE reduces the life span. The control flies already showed a difference in the life span between male and female flies. Female flies were shown to have higher average life span compared with male flies. Female flies supplemented with WAEE had an average life span comparable with that of the control group. Male flies supplemented with the low dose $(0.01 \mathrm{mg} / \mathrm{ml})$ of WAEE significantly increased the life span of flies $(p<0.05$; Fig. 3$)$. 


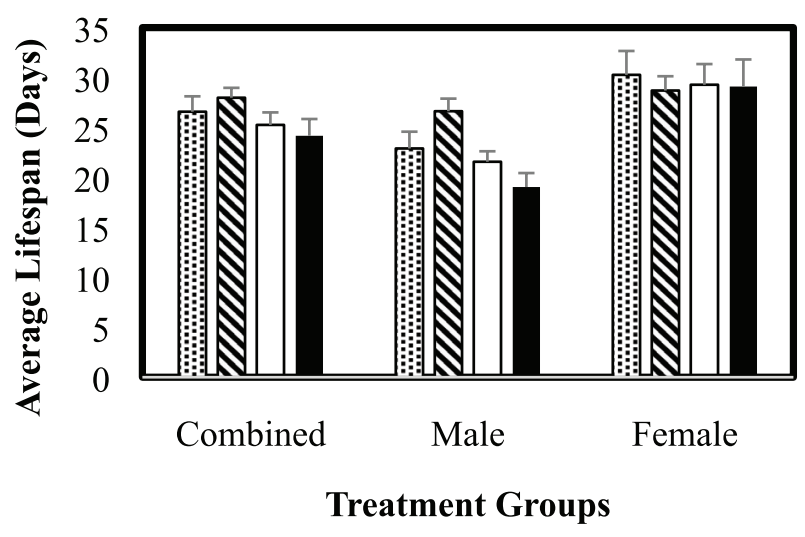

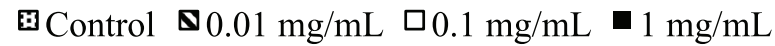

Figure 3. Life span of WAEE-supplemented D. melanogaster under normal conditions.

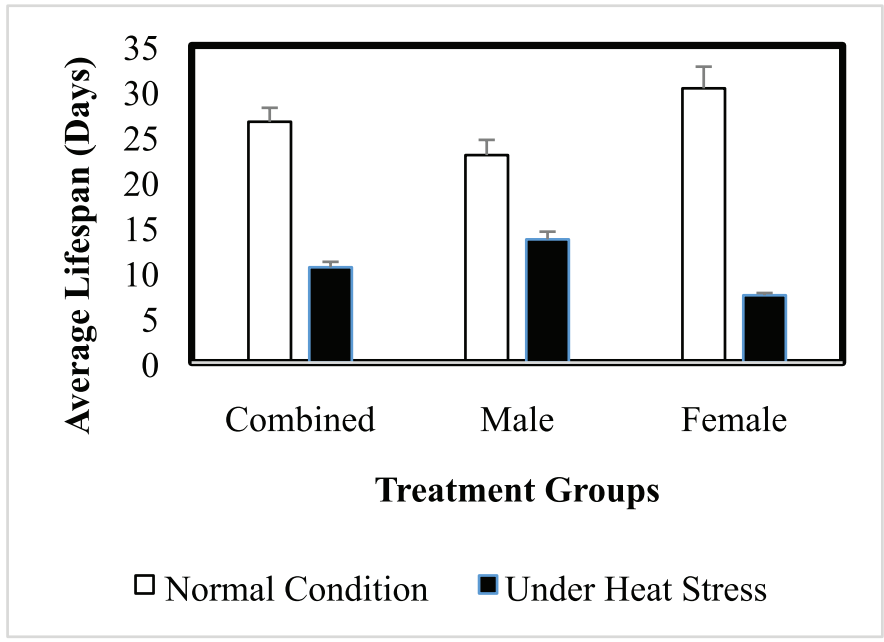

Figure 5. Life span of control flies under normal condition and heat stress condition.

Flies supplemented with CAEE had a significant life span reduction compared with the control group. The decrease in life span was more evident in female flies since all concentrations of CAEE resulted in a significant decrease in the life span. However, in male flies, only the high dose $(1 \mathrm{mg} / \mathrm{ml})$ of CAEE resulted in a significant decrease in the life span ( $p<0.05$; Fig. 4).

\section{Stress assays}

When supplemented flies were exposed to stressful conditions, the life span of male flies decreased but the life span of female flies increased. The stressful conditions were induced by subjecting flies to heat stress and starvation stress. Heat stress significantly reduced the life span of flies for both sexes; however, it was more evident in female flies under heat stress. Male flies had a $40.62 \%$ reduction in life span, while female flies had a $75.45 \%$ reduction in life span (Fig. 5).

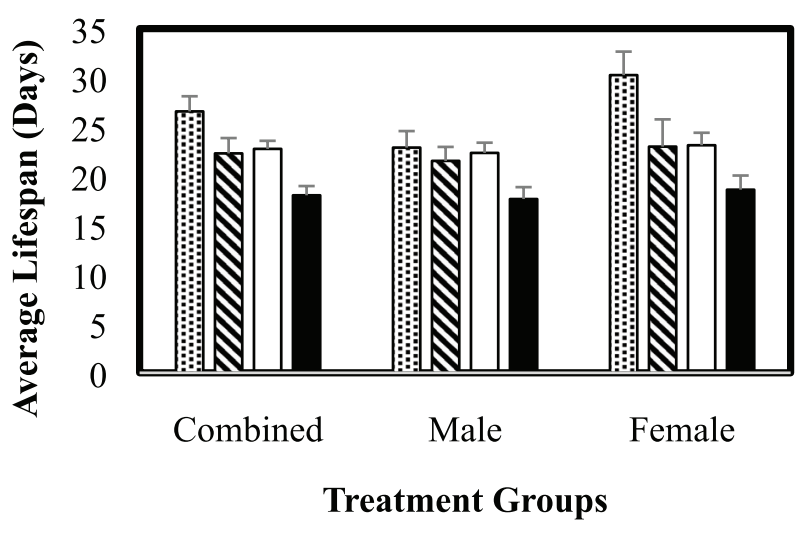

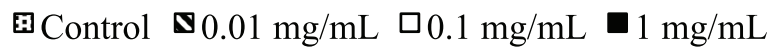

Figure 4. Life span of CAEE-supplemented D. melanogaster under normal condition.

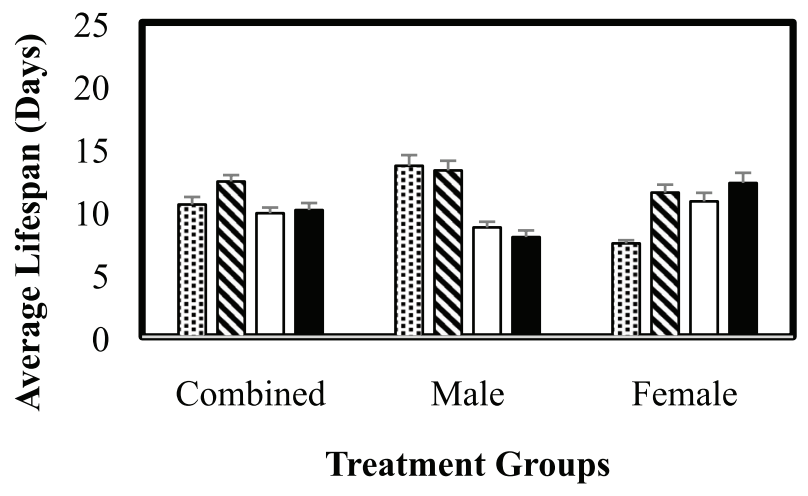

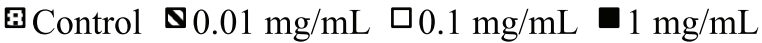

Figure 6. Life span of WAEE-supplemented D. melanogaster after the presence of daily heat stress.

Supplementation with WAEE showed a profound sexspecific effect on the life span of $D$. melanogaster under heat stress. There was an observed significant life span reduction in male flies. However, WAEE significantly preserved the life span of female flies. It even significantly increased the life span of female flies as compared with the control flies under heat stress (Fig. 6).

Supplementation with CAEE preserved the life span of male flies under heat stress and it significantly increased $(p<0.05)$ the life span of female flies across all concentrations; however, no dose-response relationship was observed (Fig. 7).

The energy deprivation significantly decreased the life span of control flies in both sexes. Reduction in life span was more evident in female flies with $52.96 \%$ reduction in life span, while there was only $29.21 \%$ reduction in the life span of male flies (Fig. 8). 


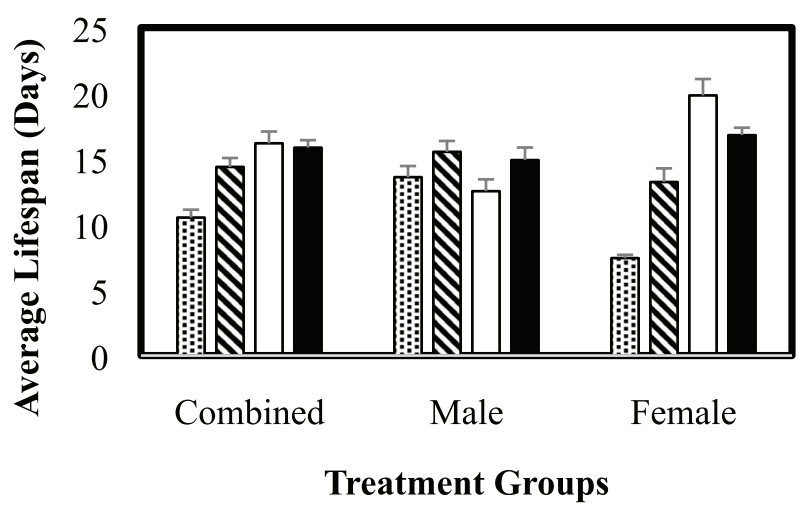

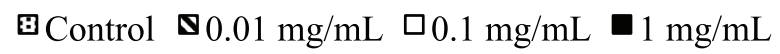

Figure 7. Life span of CAEE-supplemented D. melanogaster after the presence of daily heat stress.

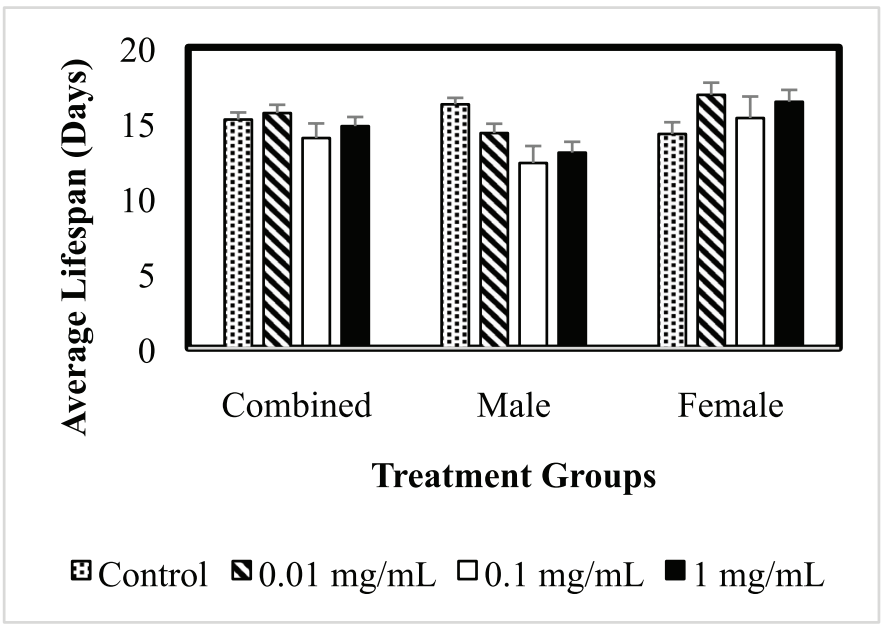

Figure 9. Life span of WAEE-supplemented D. melanogaster after starvation stress.

The supplementation with WAEE increased the life span of female flies compared with control flies (Fig. 9). However, it significantly decreased the average life span of male flies. No dose-response relationship was observed in the effects of WAEE in the life span of both male and female flies.

The supplementation with CAEE significantly decreased the life span of male flies under starvation (Fig. 10). A doseresponse relationship was observed since higher concentrations of CAEE resulted in a greater decrease in the life span. However, CAEE significantly increased the life span of female flies under starvation.

\section{Differential antioxidant activity of cultivated and wild $A$. auricula- judae ethanolic extracts}

The differences in the nutritional values and concentration of bioactive compounds may explain the differences in the antioxidant activity of wild and cultivated A. auricula-judae (Boonsong et al., 2016). These differences may cause the variances

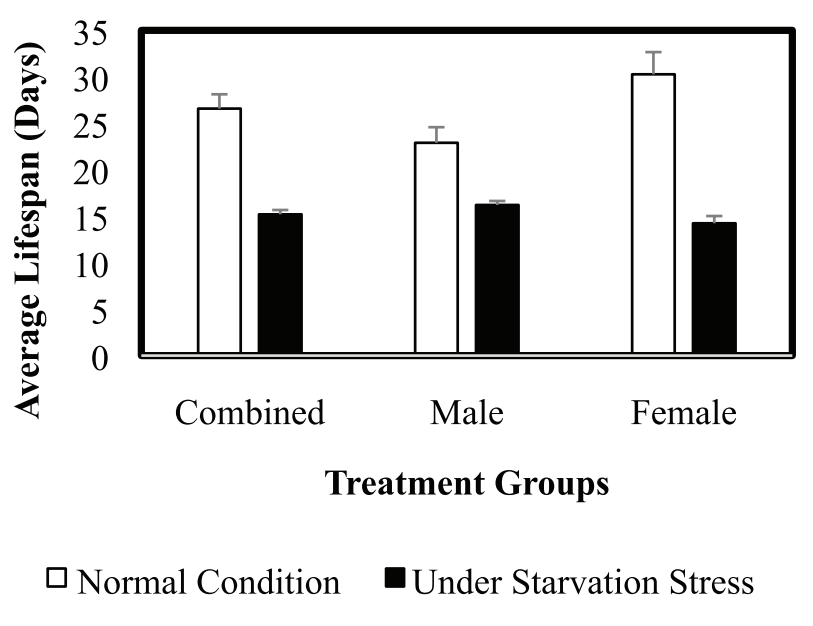

Figure 8. Life span of control flies under normal condition and energy-deprived condition.

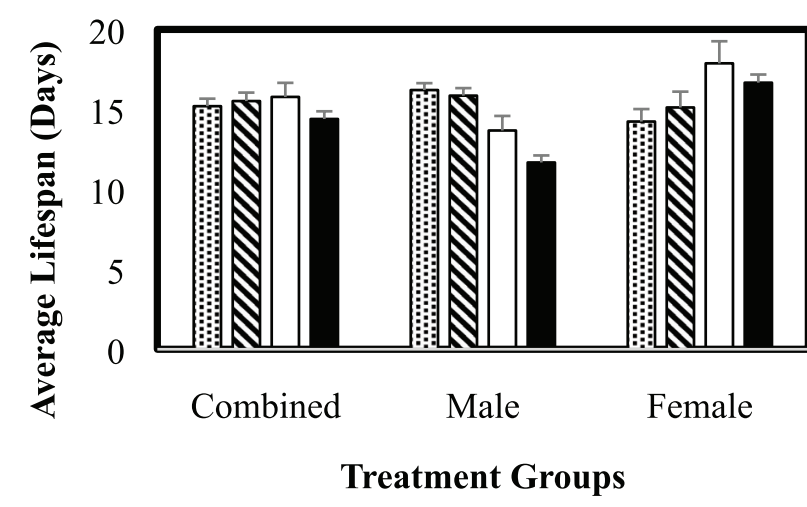

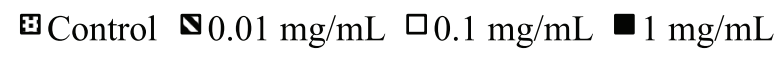

Figure 10. Life span of CAEE-supplemented D. melanogaster after starvation stress.

in the antioxidant activities of wild and cultivated mushrooms. The controlled environment of cultivated $A$. auricula-judae was seen to be beneficial in terms of antioxidant activity. The previous study also showed a higher antioxidant activity of the cultivated mushroom compared with mushroom from the wild (Wasser and Weis, 1999). These results suggested that cultivating edible mushrooms in a controlled environment did not decrease the antioxidant activity of the bioactive compounds present in the wild source. In fact, it may even enhance the antioxidant activity of the edible mushroom.

During non-stressful conditions, $A$. auricula-judae ethanolic extracts decreased the life span of flies

The results of the LSA have revealed the potential toxic effect of cultivated $A$. auricula-judae to D. melanogaster. High concentrations of CAEE and WAEE resulted in a significant decrease in the life span of male flies. WAEE did not decrease the life span of female flies while CAEE resulted in the reduction in life span. Female flies feed more frequently 


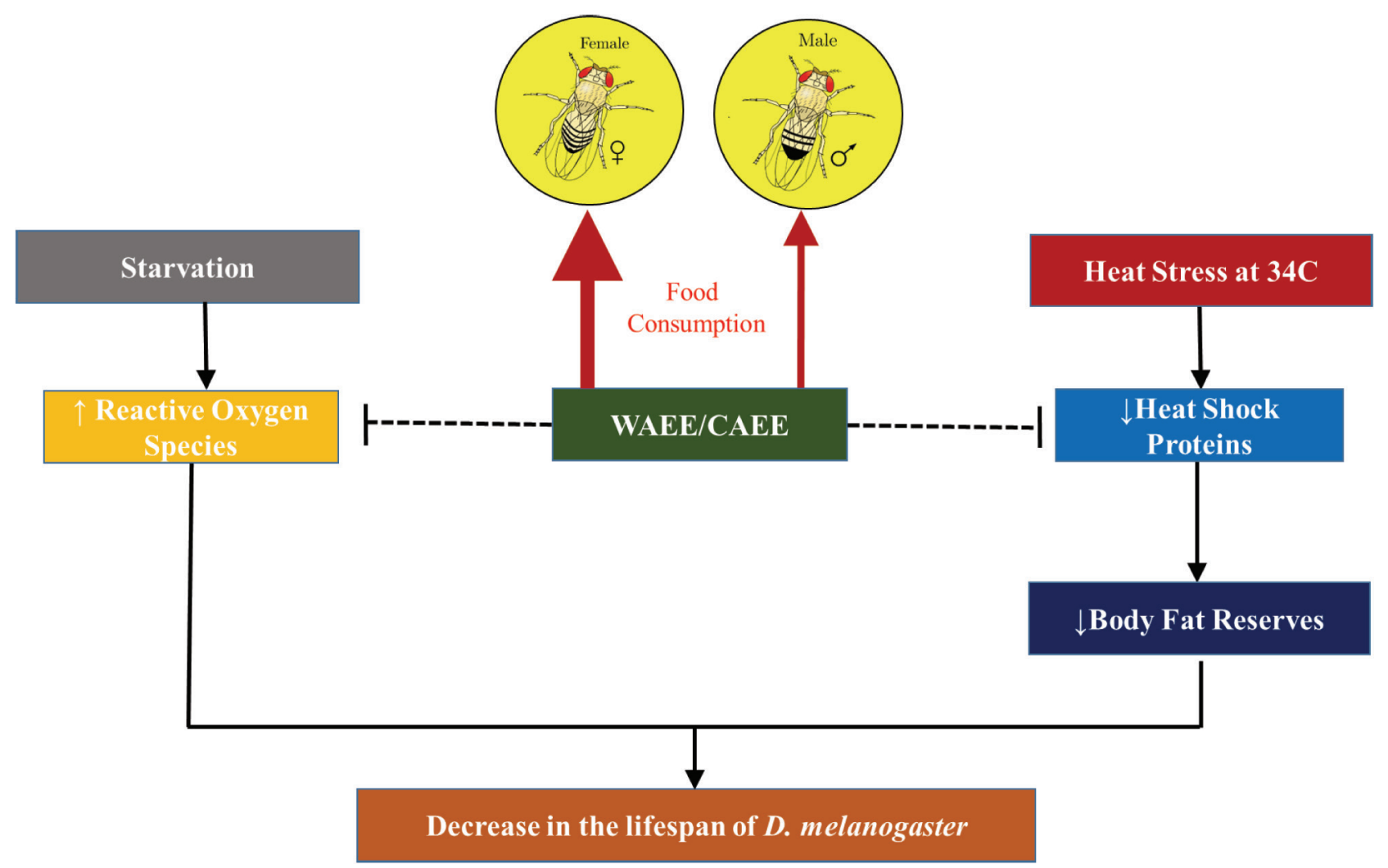

Figure 11. Proposed mechanism of action of CAEE and WAEE in the life span of D. melanogaster under normal condition and under starvation and heat stress.

than males (Wong et al., 2009). Since the antioxidant activity of CAEE is three-fold higher compared with WAEE, female flies have a higher intake of the food containing antioxidant compounds. Antioxidants at high concentration can cause prooxidative effects to the flies.

There are several antioxidants that do not increase the life span of flies (Le Bourg, 2001). It was hypothesized that several antioxidants may have pro-oxidative effects and be potentially toxic to flies if there is a failure to utilize and clear these antioxidants from the body (Velasco and Medina, 2014). Similar effects were seen in a previous study where they reported a dosage-dependent decrease in the life span of D. melanogaster (Dahomey strain) fed with exogenous antioxidants mixed with the regular diet of the flies (Magwere et al., 2004). Another possible explanation is the high levels of protein content in the mushroom extracts. The previous study observed that an increase in protein content can decrease longevity (Sanchez et al., 2015).

\section{Female flies are more susceptible to life span reduction from heat stress and starvation}

Mild heat stress (exposure to $34^{\circ} \mathrm{C}$ ) tends to increase life span more in males than in females, at least in Drosophila. One possible explanation is that female flies are more sensitive to heat stress compared with male flies. Heat resistance increased in male but only slightly in female flies (Sørensen et al., 2007). This may explain the difference in the response of male and female flies to heat stress. Female flies have a more profound decrease in their life span upon exposure to heat stress because of higher sensitivity and lower resistance to heat stress.
The same trend was observed in male and female flies under starvation. A previous study showed that female flies showed a steeper decrease in starvation resistance compared with male flies. Male flies showed a decrease in the rate of metabolite utilization and lower energy requirement to survive under starvation stress as compared to female flies. The inability to decrease metabolic rate and energy requirement in female flies resulted in the more profound decrease in the life span in female flies (Aggarwal, 2014).

Flies used in this study were all virgin flies. It was noted in a previous study that mated female flies have higher starvation resistance compared with virgin female flies; however, the absence of mating did not decrease the starvation resistance of male flies. Thus, virgin female flies are expected to be more sensitive to starvation stress compared with virgin male flies. This explained the more pronounced decrease in the life span of female flies under starvation stress compared with male flies (Goenaga et al., 2012).

Effect of CAEE and WAEE in the life span of $D$. melanogaster under normal condition and under starvation and heat stress is shown in Figure 11.

Starvation and heat stress decreased the life span of flies but the effect was more profound in female flies. Heat stress decreased heat shock proteins and body fat reserves, while starvation impaired the cellular antioxidant system which increased the levels of reactive oxygen species (ROS). These processes decreased the life span of $D$. melanogaster. The observed increase in the life span of flies treated with CAEE and WAEE may be explained by the antioxidant activity of the extracts, which can decrease the levels of ROS and enhance the levels of heat shock proteins. However, without starvation and heat stress, CAEE and 
WAEE decreased the life span of flies and this may be attributed to pro-oxidative effects of antioxidant compounds when they are not utilized and have accumulated in the body.

\section{Under starvation and heat stress, CAEE and WAEE decreased the life span of male flies but prolonged the life span of female flies}

The effect of the supplementation of WAEE and CAEE on the life span of D. melanogaster provides an additional knowledge on the understanding of the role of antioxidants in the physiology and metabolism of flies during heat stress. Exposure to high temperatures around $29^{\circ} \mathrm{C}$ and above can result to damage in heat shock proteins in flies. Heat shock response is essential to protect flies from body fat decline. Heat stress can also increase cellular stress and damage which can promote apoptosis of fat body cells. These cellular damage, decrease in body fat reserve, and damage in heat shock protein may explain the decrease in the life span of flies exposed to heat stress (Klepsatel et al., 2016). A previous study showed that the increased levels of heat shock proteins are associated with increased life span in flies (Hercus et al., 2003). Antioxidant compounds can increase the levels of heat shock proteins which can help prevent damage from oxidative stress (Geiger and Gupte, 2011). Based on these two studies, we hypothesized that the high antioxidant activity of CAEE and WAEE increased the heat shock proteins in female flies which resulted in higher average life span in female flies compared with male and control flies. This area will be the focus of future studies.

The antioxidant activity of the extract can be considered as stress enzymes. The previous studies have hypothesized that they can protect the flies during stressful conditions but they are of no use under normal conditions (Le Bourg, 2001). CAEE did not increase the life span of flies under normal conditions but it significantly increased the life span of male and female flies under starvation and heat stress. Although supplementation with WAEE and CAEE did not prevent the reduction of life span brought by the heat stress in male flies. However, flies supplemented with WAEE and CAEE still have higher average life span compared with the control flies. The presence of antioxidant compounds from WAEE and CAEE may have compensated for the decreased resistance to oxidative stress resulting to higher average life span in female flies. Since female flies feed more frequently than male flies, they have a higher intake of WAEE and CAEE containing antioxidant compounds, this may explain the greater increase in the life span of female flies.

There are reports that antioxidant enzymes help to increase the life span of flies by allowing the organisms to adapt during stressful conditions (Wong et al., 2009). Prolonged starvation can also result in oxidative stress and failure in cellular antioxidant defenses. This failure can probably be caused by inhibition of G6PDH activity. Starvation increased the ROS generation, and the antioxidant system of $D$. dentex was not able to totally scavenge the free radical which leads to lipid peroxidation (Morales et al., 2004). Since CAEE and WAEE have high antioxidant activity, it may have allowed the flies to cope with starvation stress and counter the oxidative stress from prolonged starvation. The higher life span of female flies may be due to higher intake of food supplemented with CAEE and WAEE.

\section{CONCLUSIONS}

Our data showed the sexually dimorphic effects of CAEE and WAEE in the life span of D. melanogaster under normal, starvation, and heat stress conditions. CAEE exhibited a higher antioxidant activity than WAEE in DPPH assay. Under normal condition, CAEE did not have an effect in the life span of male flies but it decreased the life span of female flies while WAEE showed no effects in the male and female flies. The life span reduction due to CAEE supplementation may be due to the pro-oxidative effects of antioxidants in female flies. Starvation and heat stress significantly decreased the life span of male and female flies. Excessive production of ROS and a decrease in heat shock proteins were hypothesized to be the cause of the observed life span reduction. Supplementation with CAEE and WAEE countered the life span reduction in female flies but not in male flies.

\section{ACKNOWLEDGMENTS}

This research did not receive any specific grant from funding agencies in the public, commercial, or not-for-profit sectors.

\section{CONFLICT OF INTEREST}

The authors declare no conflict of interest.

\section{FUNDING}

The funding sponsors had no role in the design of the study; in the collection, analyses, or interpretation of data; in the writing of the manuscript, and in the decision to publish the results.

\section{REFERENCES}

Acharya K, Yonzone P, Rai M, Acharya R. Antioxidant and nitric oxide synthase activation properties of Ganoderma applanatum. Indian J Exp Biol, 2005; 43:926-9.

Aggarwal DD. Physiological basis of starvation resistance in Drosophila leontia: analysis of sexual dimorphism. J Exp Biol, 2014; 217:1849-59.

Asatiani MD, Elisashvili V, Wasser SP, Reznick A, Nevo E. Antioxidant activity of submerged cultured mycelium extracts of higher Basidiomycetes mushrooms. Int J Med Mushrooms, 2007; 9:151-8.

Azizur Rahman M, Abdullah N, Aminudin N. Corroborative assessment of mushroom as the graceful ageing and lifespan promoting agent. Biointerface Res Appl Chem, 2017; 7(3):2072-84.

Boonsong S, Klaypradit W, Wilaipun P. Antioxidant activities of extracts from five edible mushrooms using different extractants. Agric Nat Resour, 2016; 50:89-97.

Geiger PC, Gupte AA. Heat shock proteins are important mediators of skeletal muscle insulin sensitivity. Exerc Sport Sci Rev, 2011; $39: 34-42$

Goenaga J, Mensch J, Fanara JJ, Hasson E. The effect of mating on starvation resistance in natural populations of Drosophila melanogaster. Evol Ecol, 2012; 26:813-23.

Han SK, Lee D, Lee H, Kim D, Son HG, Yang JS, Lee SJ, Kim S. OASIS 2: online application for survival analysis 2 with features for the analysis of maximal lifespan and healthspan in aging research. Oncotarget, 2016; 7:56147-52.

Hercus MJ, Loeschcke V, Rattan SIS. Lifespan extension of Drosophila melanogaster through hormesis by repeated mild heat stress. Biogerontology, 2003; 4:149-56.

Huang M, Liu J, Zhang S, Mei X, Yang X. Effects of bioactive extracts from four edible mushrooms on the lifespan of Drosophila melanogaster. Mycology, 2011; 2(1):54-8. 
Hunt PR, Son TG, Wilson MA, Yu Q, Wood WH, Becker KG, Greig NH, Mattson MP, Camandola S, Wolkow CA. Extension of lifespan in $C$. elegans by naphthoquinones that act through stress hormesis mechanisms. PLoS One, 2011; 6(7):e21922.

Iliadi KG, Boulianne GL. Age-related behavioral changes in Drosophila. Ann N Y Acad Sci, 2010; 1197:9-18.

Kadnikova IA, Costa R, Kalenik TK, Guruleva ON, Yanguo S. Chemical composition and nutritional value of the mushroom Auricularia auricula-judae. J Food Nutr Res, 2015; 3:478-82.

Kho YS, Vikineswary S, Abdullah N, Kuppusamy UR, Oh HI. Antioxidant capacity of fresh and processed fruit bodies and mycelium of Auricularia auricula-judae (Fr.) Quél. J Med Food, 2009; 12:167-74.

Klepsatel P, Gáliková M, Xu Y, Kühnlein RP. Thermal stress depletes energy reserves in Drosophila. Sci Rep, 2016; 6:1-12.

Kumar KVK, Prasanna KS, Ashadevi JS. Asparagus racemosus extract increases the life span in Drosophila melanogaster. J Appl Biol Biotechnol, 2015; 3:49-55.

Le Bourg E. Oxidative stress, aging and longevity in Drosophila melanogaster. FEBS Lett, 2001; 498:183-6.

Lin WS, Chen JY, Wang JC, Chen LY, Lin CH, Hsieh TR, Wang MF, Fu TF, Wang PY. The anti-aging effects of Ludwigia octovalvis on Drosophila melanogaster and SAMP8 mice. Age (Omaha), 2014; 36:689-703.

Luo Y, Chen G, Li B, Ji B, Guo Y, Tian F. Evaluation of antioxidative and hypolipidemic properties of a novel functional diet formulation of Auricularia auricula and Hawthorn. Innov Food Sci Emerg Technol, 2009; 10:215-21.

Magwere T, Chapman L, Partridge L. Sex differences in the effect of dietary restriction on life span and mortality rates in female and male Drosophila melanogaster. J Gerontol A Biol Sci Med Sci, 2004; 59:3-9.

Morales AE, Pérez-Jiménez A, Carmen Hidalgo M, Abellán E, Cardenete G. Oxidative stress and antioxidant defenses after prolonged starvation in Dentex dentex liver. Comp Biochem Physiol C Toxicol Pharmacol, 2004; 139:153-61.

Rawal S, Singh P, Gupta A, Mohanty S. Dietary intake of curcuma longa and Emblica officinalis increases life span in Drosophila melanogaster. Biomed Res Int, 2014; 2014: Article ID 910290.
Sanchez JE, Jimenez-Perez G, Liedo P. Can consumption of antioxidant rich mushrooms extend longevity?: antioxidant activity of Pleurotus spp. and its effects on Mexican fruit flies? (Anastrepha ludens) longevity. Age (Omaha), 2015; 37:1-10.

Sørensen JG, Kristensen TN, Kristensen KV, Loeschcke V. Sex specific effects of heat induced hormesis in Hsf-deficient Drosophila melanogaster. Exp Gerontol, 2007; 42:1123-9.

Sun Y, Yolitz J, Wang C, Spangler E, Zhan M, Zou S. Aging studies in Drosophila Melanogaster., In: Tollefsbol, T (ed.). Biologica aging. Methods in molecular biology (methods and protocols). Humana Press, Totowa, NJ, pp 77-93, 2013.

Velasco AJD, Medina PMB. Crude anthocyanin extract (CAE) from ballatinao black rice does not alter longevity and increases stress susceptibility of Drosophila melanogaster. Int J Curr Res Biosci Plant Biol, 2014; $1: 35-42$

Wasser SP, Weis AL. Therapeutic effects of substances occurring in higher Basidiomycetes mushrooms: a modern perspective. Crit Rev Immunol, 1999; 19:65-96.

Wong R, Piper MDW, Wertheim B, Partridge L. Quantification of food intake in Drosophila. PLoS One, 2009; 4:1-10.

Zou Y, Hu W, Ma K, Tian M. Physicochemical properties and antioxidant activities of melanin and fractions from Auricularia auricula fruiting bodies. Food Sci Biotechnol, 2015; 24:15-21.

\section{How to cite this article:}

Romero JJN, Ramos MRRE, Tantengco OAG, Medina PMB. Sex differences in the effects of Auricularia auriculajudae ethanolic extracts on the life span of Drosophila melanogaster during stress and non-stress conditions. J App Pharm Sci, 2018; 8(11): 087-094. 